$\mathrm{BNL}-67823$

CAP-292-Muon-00C

\title{
INTENSE MUON BEAMS AND NEUTRINO FACTORIES
}

Zohreh Parsa

Brookhaven National Laboratory, New York

October 2000

$+4$ 


\title{
INTENSE MUON BEAMS AND NEUTRINO FACTORIES
}

\author{
Zohreh Parsa \\ Brookhaven National Laboratory $(B N L)^{*}$ \\ Physics Department 510 A, \\ Upton, NY 11973-5000, USA
}

\begin{abstract}
High intensity muon sources are needed in exploring neutrino factories, lepton flavor violating muon processes, and lower energy experiments as the stepping phase towards building higher energy $\mu^{+} \mu^{-}$colliders. We present a brief overview, sketch of a neutrino source, and an example of a muon storage ring at BNL with detector(s) at Fermilab, Sudan, etc. Physics with low energy neutrino beams based on muon storage rings ( $\mu \mathrm{SR})$ and conventional Horn Facilities are described and compared. $\mathrm{CP}$ violation Asymetries and a new Statistical Figure of Merit to be used for comparison is given. Improvements in the sensitivity of low energy experiments to study Flavor changing neutral currents are also included.
\end{abstract}

\section{INTRODUCTION}

There is a great deal of interest in high-intensity muon beams and their use in high luminosity muon colliders, neutrino factories, rare interactions and decays of muons. The theoretical interest to explore lepton flavor violating muon processes is motivated in part by supersymmetric grand unified models of particle interactions and ideas to make substantial improvements in the sensitivity of experiments to study rare muon processes.

A muon collider requires as its starting point, a very intense beam of muons with a small momentum spread. Such beams would be accelerated to collider energies and be used to search for new short distance high energy phenomena. A muon storage ring based neutrino factory is a natural path to muon collider technology, since both facilities share essentially the same subcomponents prior to the storage ring. In section 2, we briefly describe the physics and production of high-intensity muon and neutrino beams. An example of a neutrino source at BNL is described in section 3 , and a comparison of conventional Horn beam and muon storage produced

*) Supported by US Department of Energy contract DE-AC02-98CH10886. Accordingly, the U.S. Government retains a non-exclusive, royalty-free license to publish or reproduce the published form of this contribution, or allow others to do so, for U.S. Government purposes.

†) E-mail: parsa@bnl.gov 
beam are given in section 4 . In addition, a survey of low energy muon physics including muon number non-conservation, and current bounds are given in section 5 , a summary in section 6 and references in section 7 .

\section{INTENSE MUON BEAMS - NEUTRINO SOURCES - PHYSICS}

A muon storage ring based Neutrino Source (Neutrino Factory) beside providing a first phase of a muon collider facility, it would generate more intense and well collimated neutrino beams than currently available. The BNL-AGS or some other proton driver would provide an intense proton beam that hits a target, produces pions that decay into muons. The muons must be cooled, accelerated and injected into a storage ring with a long straight section where they decay. The decays occurring in the straight sections of the ring would generate neutrino beams that could be directed to detectors located thousands of kilometers away. allowing studies of neutrino oscillations with precisions not currently accessible.

The composition and spectra of an intense neutrino beam from a muon storage ring depends on momentum, polarization and charge of the stored muons, through the decays $\mu^{-} \rightarrow e^{-} \nu_{\mu} \bar{\nu}_{e}$ or $\mu^{+} \rightarrow e^{+} \bar{\nu}_{\mu} \nu_{e}$.

Table 1 illustrates (a summary of the standard model). spectrum of neutrinos with some of their basic properties. The fermions are grouped into three generations of spin $1 / 2$ leptons and quarks which span an enormous mass range.

The idea of muon storage rings has been discussed since at least 1960 [1]. However, storage rings with enough circulating muons to provide higher intensity neutrinos than from conventional horn beams have only been considered more recently,

TABLE 1. Neutrinos and Their Properties

\begin{tabular}{lccccc}
\hline Symbol & Spin & Charge & Color & Mass $(\mathrm{GeV})$ & \\
\hline$\nu_{e}$ & $1 / 2$ & 0 & 0 & $<4.5 \times 10^{-9}$ & \\
$e$ & $1 / 2$ & -1 & 0 & $0.51 \times 10^{-3}$ & First \\
$u$ & $1 / 2$ & $2 / 3$ & 3 & $5 \times 10^{-3}$ & Generation \\
$d$ & $1 / 2$ & $-1 / 3$ & 3 & $9 \times 10^{-3}$ & \\
& & & & & \\
$\nu_{\mu}$ & $1 / 2$ & 0 & 0 & $<.16 \times 10^{-3}$ & \\
$\mu$ & $1 / 2$ & -1 & 0 & 0.106 & Second \\
$c$ & $1 / 2$ & $2 / 3$ & 3 & 1.35 & Generation \\
$s$ & $1 / 2$ & $-1 / 3$ & 3 & 0.175 & \\
& & & & & \\
$\nu_{\tau}$ & $1 / 2$ & 0 & 0 & $<2.4 \times 10^{-3}$ & \\
$\tau$ & $1 / 2$ & -1 & 0 & 1.775 & Third \\
$t$ & $1 / 2$ & $2 / 3$ & 3 & $174.3 \pm 5.1$ & Generation \\
$b$ & $1 / 2$ & $-1 / 3$ & 3 & 4.5 & \\
\hline
\end{tabular}


in the context of muon collider technology [7].

The neutrino fluxes from the proposed muon-based beams would be higher than ever previously achieved with a much better-understood flavor composition. In addition, since the neutrino beams from these sources would be secondary beams from high energy muon decays, they would be extremely well collimated. Distances between production and detection could, therefore span the globe. Using the precisely known flavor composition of the beam, one could envision an extensive program to measure the neutrino oscillation mixing matrix, including possible $\mathrm{CP}$ violating effects. The number of neutrino interactions per unit mass of a detector at distance $L$ from a muon storage ring operating at energy $E_{\mu}$ scales as

$$
N_{\text {events }} \propto N_{\mu} E_{\mu}^{3} L^{-2}
$$

for the example of a proton source with $1.5 \mathrm{MW}$ power, in one year $\left(10^{7} \mathrm{~s}\right)$ of operation, there would be about $4 \times 10^{20}$ muons per year decaying in the storage ring. Assuming the fraction of the ring pointing to a given detector to be about 0.25 (as in example of a bowtie-shaped muon storage) then the number of decays pointing to the given detector will be approximately $10^{20}$. It may be noted that the number of events with the 1.5 MW neutrino factory, in a detector at the same $730 \mathrm{~km}$, is approximately 100 times that in the proposed CERN - Gran Sasso experiment (NGS) [9], and about 40 times the maximum event rate that MINOS [10] can expect. Upgrading the proton driver to $4 \mathrm{MW}$, the factors become about 300 and 100 for Gran Sasso and Soudan, respectively.

At the detectors, the neutrino and the antineutrino may or may not have changed their flavor, leading to the appearance of a different flavor or the disappearance of the initial flavor, respectively. When detected by a charged-current interaction, there are 6 classes of signatures in a three-neutrino model:

1) $\nu_{\mu} \rightarrow \nu_{e} \rightarrow e^{-}$(appearance);

2) $\nu_{\mu} \rightarrow \nu_{\mu} \rightarrow \mu^{-}$(disappearance);

3) $\nu_{\mu} \rightarrow \nu_{\tau} \rightarrow \tau^{-}$(appearance);

4) $\bar{\nu}_{e} \rightarrow \bar{\nu}_{e} \rightarrow e^{+}$(disappearance);

5) $\bar{\nu}_{e} \rightarrow \bar{\nu}_{\mu} \rightarrow \mu^{+}$(appearance);

6) $\bar{\nu}_{e} \rightarrow \bar{\nu}_{\tau} \rightarrow \tau^{+}$(appearance).

For operation with positive muons, a similar list of processes may be written. The 5 th process where a muon of different sign from the parent muon appears, has a very unique possibilities at a neutrino factory based on muon storage rings. Since they are the only sources of intense high energy electron (anti)neutrino beams. The $\tau$ appearance (cases 3 and 6 ) are practical only for neutrino beams with 10 's of $\mathrm{GeV}$ energy.

Muon Storage rings of e.g., $E_{\mu}=50,20,10,1 \mathrm{Gel}$ have been considered and all else equal, higher energy is better (but the cost may be higher?). The high 
cost estimates of a $50 \mathrm{GeV}$ ring has led to recent studies of lower energy rings. Experiments carried out at a neutrino factory within the next decade can add compelling new information to our understanding of neutrino oscillations, if the number of useful muon decays exceeds $10^{19}$ per year, and energy is $\gtrsim 20 \mathrm{GeV}$.

\section{Neutrino Source at BNL}

As known, the BNL-AGS proton beam parameters are very suited for use as a source for muon storage ring based neutrino factory and muon collider. Table 2 illustrates basic BNL-AGS proton beam properties.

With a muon storage ring - neutrino source at BNL (Fig. 1), detectors at Fermilab or Soudan, Minnesota $(1715 \mathrm{~km})$, become very interesting possibilities. The feasibility of constructing and operating such a muon-storage-ring based NeutrinoFactory, including geotechnical questions related to building non-planar storage rings (e.g. for BNL-fermilab; at $8^{\circ}$ angle for BNL-Soudan, and $31^{\circ}$ angle for BNLGran Sasso) along with the design of the muon capture, cooling, acceleration, and storage ring for such a facility is being explored by our growing Neutrino Factory and Muon Collider Collaboration (NFMCC), but requires additional studies for a BNL site specific example.

Conventionally, neutrino beams employ a proton beam on a target to generate pions, which are focused and allowed to decay into neutrinos and, muons [10]. The muons are stopped in the shielding, while the muon-neutrinos are directed toward the detector. In a neutrino factory, pions are made the same way and allowed to decay, but it is the decay muons that are captured and used. The initial neutrinos from pion decay are discarded, or used in a parasitic low-energy neutrino experiment. But the muons are accelerated and allowed to decay in a storage ring with long straight sections. It is the neutrinos from the decaying muons (both muon-neutrinos and anti-electron-neutrinos) that are directed to a detector.

Figure 1 shows schematics of space angles [16] and baselines for example of a muon storage neutrino source at BNL, with detectors (placed at Fermilab; Soudan; Minnesota $(1715 \mathrm{~km})$; or Gran Sasso, Italy $(6527 \mathrm{~km})$ ) at various global locations.

In a Neutrino Factory, a proton driver of moderate energy $(<50 \mathrm{GeV})$ and high

TABLE 2. BNL- AGS Proton Beam Properties

\begin{tabular}{lcc}
\hline Parameters & BNL-AGS & Muon Collider \\
\hline Proton Energy [GeV] & 24 & $16-24$ \\
Proton/Bunch & $1.6 \times 10^{13}$ & $5 \times 10^{13}$ \\
Bunch No. & 6 & 2 \\
Proton/cycle & $1.0 \times 10^{14}$ & $1.0 \times 10^{14}$ \\
Bunch Length $[\mu s]$ & 2.2 & 1 \\
Bunch spacing [ns] & 440 & 1000 \\
\hline
\end{tabular}



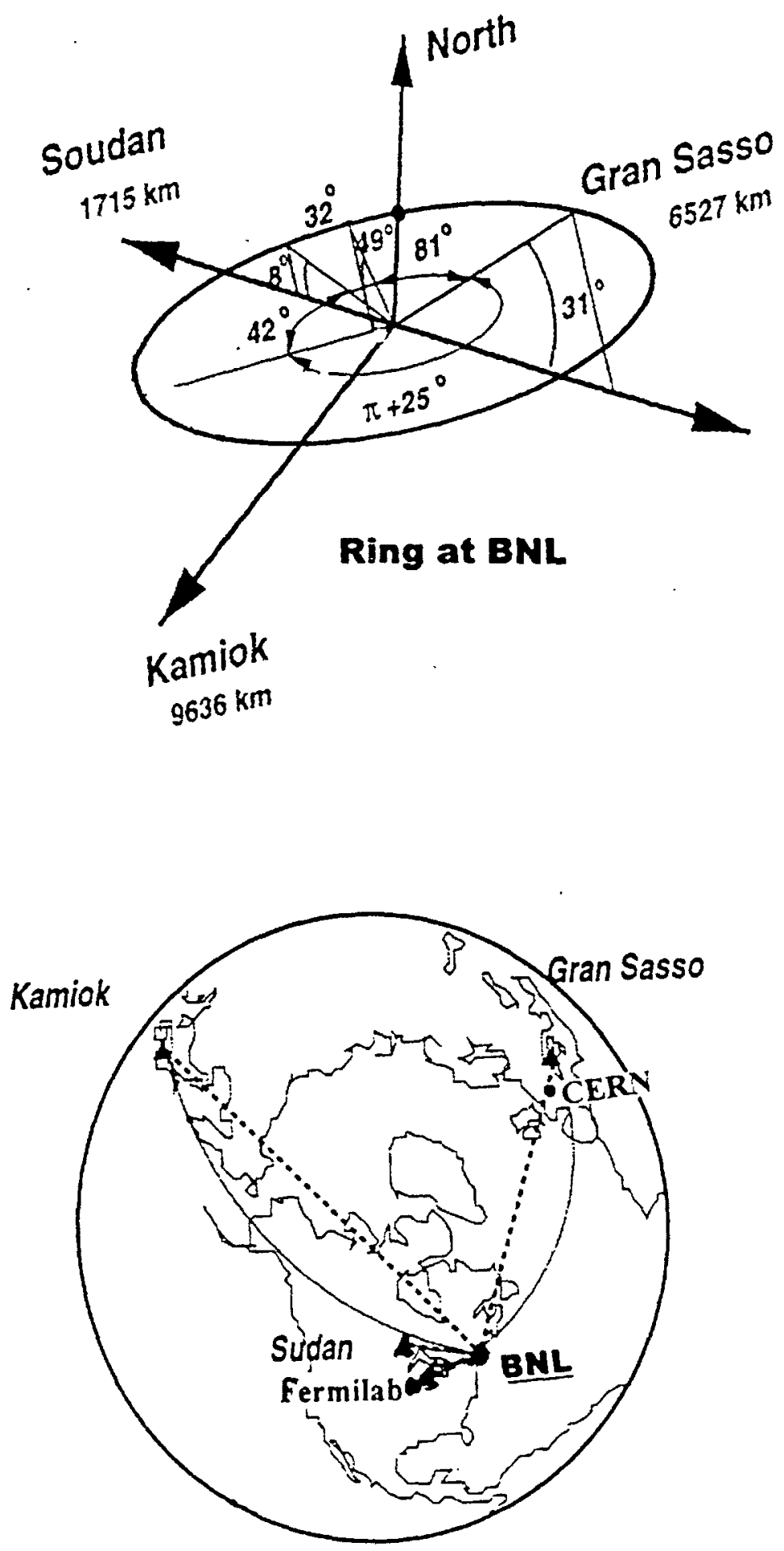

FIGURE 1. Shows space angles and baselines for a Muon - Storage Ring at BNL and possible detector sites (at Fermilab, Sudan, CERN, Kamioka and Gran Saso). 
average power,(e.g., 1-4 MW), similar to that required for a muon collider, but with a less stringent requirements on the charge per bunch and power is needed. This is followed by a target and a pion-muons capture system. A longitudinal phase rotation is performed to reduce the muon energy spread at the expense of spreading it out over a longer time interval. The phase rotation system may be designed to correlate the muon polarization with time, allowing control of the relative intensity of muon and anti-electron neutrinos. Some cooling may be needed, to reduce phase space, about a factor of $50 \mathrm{in} \mathrm{six} \mathrm{dimensions.} \mathrm{This} \mathrm{is} \mathrm{much} \mathrm{smaller} \mathrm{than} \mathrm{the} \mathrm{factor} \mathrm{of}$ $10^{6}$ needed for a muon collider. Production is followed by fast muon acceleration to $50 \mathrm{GeV}$ (for example), in a system of linac and two recirculating linear accelerators (RLA's), which may be identical to that for a first stage of muon collider such as a Higgs Factory. A muon-storage ring with long straight sections could point to one or more distant neutrino detectors for oscillation studies, and to one or more near detectors for high intensity scattering studies.

A planar bowtie - shaped ring can be designed and oriented to send neutrino beams to any two detector sites. Since, there is no net bending, the polarization may be preserved. (A disadvantage of the Bowtie - shaped ring is that it may need extra bending. Since there is geometry constrains on the ratio of short to long straight sections, the ring circumference may increase.) With the ring in a tilted plane, both long straight sections would point down into the earth, such that neutrinos can be directed into two very distant detectors. Triangular-shaped storage rings also have this advantage.

Figure 2 illustrates a schematic concept of a Neutrino Factory Facility based on a bowtie muon storage lattice. The examples described are based on some of the scenarios being explored by our NFMCC, [4].

A neutrino factory has a strong independent physics case. It would be easier to build, less expensive than a full muon collider, and could demonstrate most of the components of a collider.

Table 3, gives charged current neutrino interaction rates (per kiloton-year) as a function of baseline length $L$ for an $E_{\mu}=50 \mathrm{GeV}$ muon storage ring in which there are $1 \times 10^{20}$ unpolarized muon decavs per year within a neutrino beam-forming straight section [17]. The rates are listed for oscillations:

1) $\nu_{e} \rightarrow \nu_{\mu}: \Delta m^{2}=3.5 \times 10^{-3} \mathrm{eV}^{2} / \mathrm{c}^{4} \& \sin ^{2} 2 \theta=0.1$,

2) $\nu_{e} \rightarrow \nu_{\mu}: \Delta m^{2}=1 \times 10^{-4} \mathrm{el}^{2} / \mathrm{c}^{4} \& \sin ^{2} 2 \theta=1$,

3) $\nu_{e} \rightarrow \nu_{\tau}: \Delta m^{2}=3.5 \times 10^{-3} \mathrm{eV}^{-2} / \mathrm{c}^{4} \& \sin ^{2} 2 \theta=0.1$,

4) $\nu_{\mu} \rightarrow \nu_{\tau}: \Delta m^{2}=3.5 \times 10^{-3} \mathrm{eV}^{2} / \mathrm{c}^{4} \& \sin ^{2} 2 \theta=1$.

The rates for the unoscillated neutrino interactions, the corresponding statistical significance of the disappearance signal (numbers in parenthesis). and the rates for the antineutrino interactions, are also included in Table 3.

If future experiments confirm the interpretation of the LSND data that there exist more than three light neutrinos, then use of the neutrino factory flavor-rich 


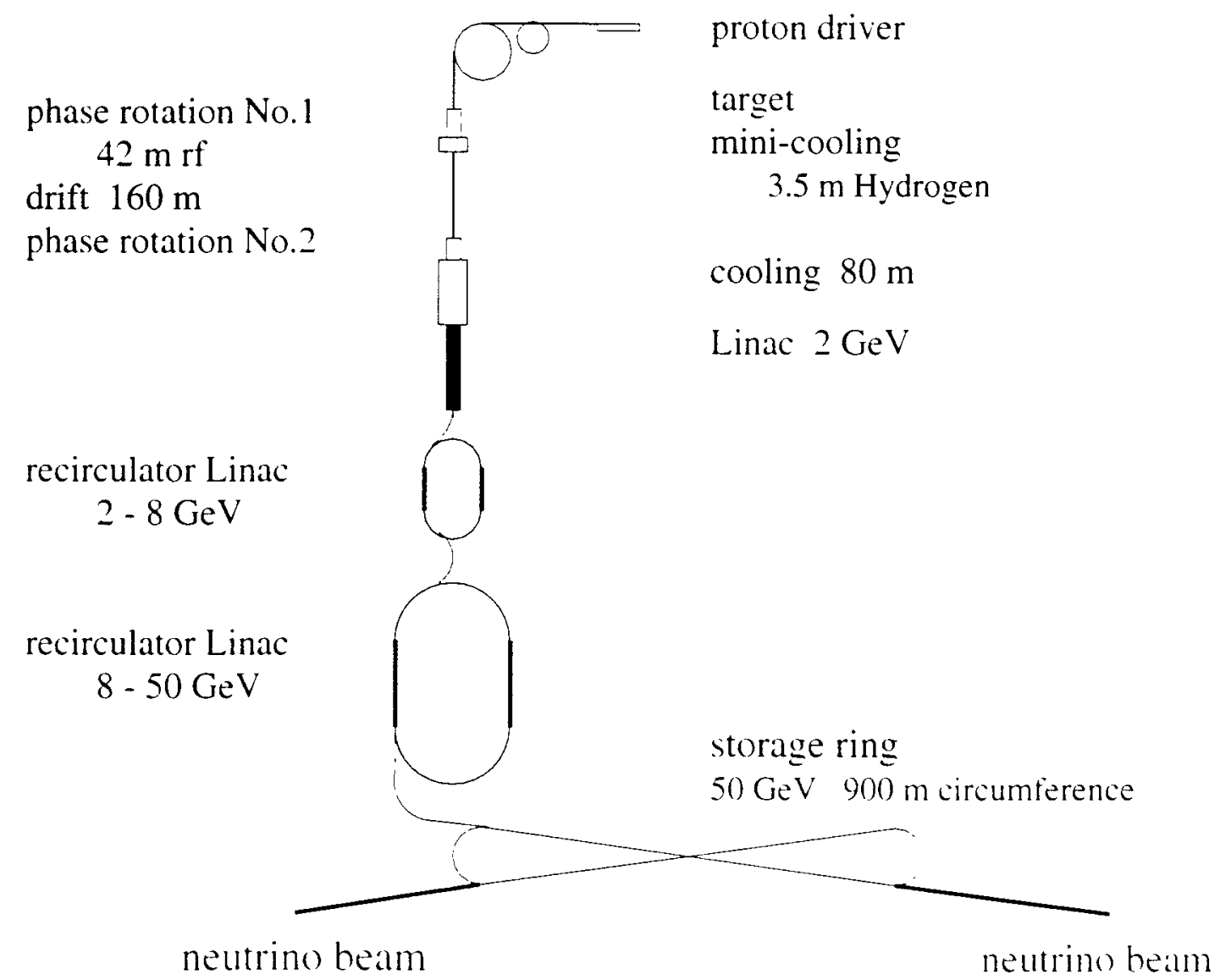

FIGURE 2. Overview of a Neutrino Factory Concept, with a Bowtie shaped Muon - Storage lattice 
TABLE 3. Neutrino Interaction Rates at a Neutrino Factory.

\begin{tabular}{|c|c|c|c|c|c|}
\hline & & $\begin{array}{c}\text { Source at } \\
\text { Detector at } \\
L(\mathrm{~km})\end{array}$ & $\begin{array}{c}\text { BNL } \\
\text { G. Sasso } \\
6527\end{array}$ & $\begin{array}{c}\text { BNL } \\
\text { SLAC } \\
4139 \\
\end{array}$ & $\begin{array}{c}\text { BNL } \\
\text { Soudan } \\
1715\end{array}$ \\
\hline $\begin{array}{c}\text { Case } \\
1 \text { ) }\end{array}$ & $\mu^{+}$ & $\begin{array}{c}\text { Mode } \\
\nu_{e} \rightarrow \nu_{\mu} \\
\nu_{e} \rightarrow \nu_{e} \\
\bar{\nu}_{\mu} \rightarrow \bar{\nu}_{\mu}\end{array}$ & $\begin{array}{c}90 \\
1400 \\
(2.4 \sigma) \\
890 \\
\end{array}$ & $\begin{array}{c}160 \\
3600 \\
(2.7 \sigma) \\
2200 \\
\end{array}$ & $\begin{array}{c}190 \\
16000 \\
(1.5 \sigma) \\
9300 \\
\end{array}$ \\
\hline 2) & $\mu^{+}$ & $\begin{array}{c}\nu_{e} \rightarrow \nu_{\mu} \\
\nu_{e} \rightarrow \nu_{e} \\
\bar{\nu}_{\mu} \rightarrow \bar{\nu}_{\mu}\end{array}$ & $\begin{array}{c}5 \times 10^{-2} \\
1500 \\
890 \\
\end{array}$ & $\begin{array}{c}0.86 \\
3800 \\
2200 \\
\end{array}$ & $\begin{array}{c}1.5 \\
16000 \\
9400 \\
\end{array}$ \\
\hline 3) & $\mu^{+}$ & $\begin{array}{l}\nu_{e} \rightarrow \nu_{\tau} \\
\nu_{e} \rightarrow \nu_{e} \\
\bar{\nu}_{\mu} \rightarrow \bar{\nu}_{\mu}\end{array}$ & $\begin{array}{c}31 \\
1400 \\
(2.4 \sigma) \\
890 \\
\end{array}$ & $\begin{array}{c}60 \\
3700 \\
(2.7 \sigma) \\
2200 \\
\end{array}$ & $\begin{array}{c}70 \\
1.6 \times 10^{4} \\
(1.5 \sigma) \\
9400\end{array}$ \\
\hline 4) & $\mu^{-}$ & $\begin{array}{l}\nu_{\mu} \rightarrow \nu_{\tau} \\
\nu_{\mu} \rightarrow \nu_{\mu} \\
\bar{\nu}_{e} \rightarrow \bar{\nu}_{e}\end{array}$ & $\begin{array}{c}450 \\
760 \\
(35 \sigma) \\
770\end{array}$ & $\begin{array}{c}570 \\
3100 \\
(23 \sigma) \\
1900\end{array}$ & $\begin{array}{c}650 \\
1.7 \times 10^{4} \\
(12 \sigma) \\
8100\end{array}$ \\
\hline
\end{tabular}

beams would be even more crucial, because the parameter space for $\mathrm{CP} / \mathrm{T}$ violating effects would be considerably enlarged and could be explored in experiments with such beams [20].

\section{LOW ENERGY $\nu$ FACTORIES AND CONVENTIONAL HORN BEAMS}

An alternative source of intense muons are the conventional Horn Beams (very efficient) which seems to be not only competitive with the lower energy muon storage rings $(\mu \mathrm{SR})$ but also at a lower cost. Low energy neutrino physics is a very competitive way to study neutrino oscillations. E.g., with $\nu_{\mu} \rightarrow \nu_{e}$ or $\nu_{e} \rightarrow \nu_{\mu}$, measure $\sin ^{2} 2 \theta_{13}$, CP violation, etc. [15]:

CP Violation Asymetries

$$
\begin{aligned}
A(L)_{C P} & \equiv \frac{P(L)_{\nu_{e} \rightarrow \nu_{\mu}}-P(L)_{\bar{\nu}_{e} \rightarrow \bar{\nu}_{\mu}}}{P(L)_{\nu_{e} \rightarrow \nu_{\mu}}+P(L)_{\bar{\nu}_{e} \rightarrow \bar{\nu}_{\mu}}} \\
& =\frac{P(L)_{\nu_{\mu} \rightarrow \nu_{e}}-P(L)_{\bar{\nu}_{\mu} \rightarrow \bar{\nu}_{e}}}{P(L)_{\nu_{\mu} \rightarrow \nu_{e}}+P(L)_{\bar{\nu}_{\mu} \rightarrow \bar{\nu}_{e}}} .
\end{aligned}
$$

Statistical Figure of Merit (F)

$$
F=\frac{A^{2}(N+\overline{\mathrm{V}})}{1-A^{2}}
$$


which grows with $E_{\nu}$ if all else is left equal. Where $N=\#$ of $\nu_{e} \rightarrow \nu_{\mu}, \bar{N}=\#$ of $\bar{\nu}_{e} \rightarrow \bar{\nu}_{\mu}, A=\frac{N-\bar{N}}{N+\bar{N}}$, and $\frac{\delta A}{A}= \pm \frac{\sqrt{1-A^{2}}}{A \sqrt{(N+\bar{N})}}$.

For example, using the BNL- AGS $E_{p} \simeq 28 \mathrm{GeV}$ if one considers the BNL P889 study [14], and increase the number of proton on target (p.o.t.) e.g., from $2 \times$ $10^{20}$ p.o.t./yr to $6 \times 10^{21}$ p.o.t./yr one gains a factor of $30, E_{\nu_{\mu}}^{\text {peak }} \simeq 1 \mathrm{GeV}$, with $\nu_{\mu} \rightarrow \mu^{-}$, at $L=1 \mathrm{~km}$ no oscillation,

$N_{\mu^{-}} \simeq 4.43 \times 10^{-15} / k T$ on $/$ p.o.t. $\times 6 \times 10^{21}$ p.o.t. $/ y r \simeq 2.7 \times 10^{7} /$ kTon $/ y r$, (Fiducial Mass $\rightarrow 1 / 2$ ).

This is Comparable to a muon storage ring with $E_{\mu} \simeq 10 \mathrm{GeV}, 2 \times 10^{20} \mu$ decays $/ y r$, with $L=10 \mathrm{~km} ; \nu_{\mu} \rightarrow \mu^{-}$or $\bar{\nu}_{e} \rightarrow e^{+}$; and $N_{\mu^{-}} \simeq 1.5 \times 10^{7} / \mathrm{kTon} / \mathrm{yr}$.

That is, (we have about the same event rates), with the same p.o.t. and detector size, $1 \mathrm{GeV} \nu_{\mu}^{\text {peak }}$ Horn $\simeq 10 \mathrm{GeV} \mu$ Storage Ring (statistically, if $L / E$ is fixed). With upgraded proton source (e.g., $6 \times 10^{14}$ p.o.t./yr ), larger detectors (e.g., $45 k T$ Ton, (preferable $>500 k$ Ton)), at about $300 \mathrm{~km}$, with low energy $\nu_{\mu}$ and $\bar{\nu}_{\mu}$ from a Horn (e.g. 300 times the BNL-P889 study) one should measure $\theta_{13}$, $\theta_{23}$ (High precision), $\Delta m^{2}{ }_{31}$ and will have some CP violation capability (e.g., one should measure $\sin ^{2} 2 \theta_{13} \gtrsim 0.007$ which is impressive).

If we increase L to 10 or 100 times longer, in the example of the BNL-P889 study with Detectors at $1,3,24,68 \mathrm{~km}$, then with 340 times the P889 event rate at $L \simeq 260 \mathrm{~km}$ most of $\nu_{\mu} \rightarrow \nu_{\tau}\left(\Delta m_{31}^{2} \simeq 3 \times 10^{-3} \mathrm{eV}^{2}\right)$, number of $\nu_{\mu} \rightarrow \nu_{e} \rightarrow e^{-}$, $\left(5 \times 10^{4} \sin ^{2} \theta_{23} \sin ^{2} 2 \theta_{13} \simeq 175\right.$ events, $\left.\left(\sin ^{2} 2 \theta_{13} \gtrsim 0.007\right)\right)$, and $3 \sigma$ measurements of $A_{C P} \simeq 0.15 \pm 0.05$. Upgraded $\nu_{\mu}, \bar{\nu}_{\mu}$ Horn Facility potentially is powerful [15]. However, there are some questions (targetry R\&D issues) that needs to be addressed. E.g., how high intensity can a conventional horn tolerate? How about using a solenoid magnet as a focusing device? Solenoid may be able to handle the high intensity proton currents better?

Similar upgrades for MINOS may be possible. E.g., a followup efforts (MINOSII), could include doing muon-electron neutrino oscillations and CP violation by (upgrading the Fermilab $8 \mathrm{GeV}$ Booster) intensifying their beam and doing low energy option.

Stop $\pi^{+} \rightarrow \nu_{\mu} \nu_{e} \bar{\nu}_{\mu} \quad E_{\mu} \simeq 30 \mathrm{MeV}$ (LSND, KARMEN) and Neutron Spallation Sources are also alternatives that may be considered but will not be discussed here.

\section{LOW ENERGY MUON PHYSICS - A SURVEY}

Using intense muon beams to carry forefront low energy research include the search for muon - number non - conservation, such as

$$
\begin{aligned}
& \mu^{+} \rightarrow e \gamma \\
& \mu^{+} \rightarrow e^{+} e^{-} e^{+} \\
& \mu^{-} N \rightarrow e^{-} N
\end{aligned}
$$


Other processes could include

$$
\begin{aligned}
& \mu^{-} N \rightarrow e^{+} N^{\prime} \\
& \mu^{-} N \rightarrow \mu^{+} N^{\prime} \\
& \mu^{+} e^{-} \rightarrow \mu^{-} e^{+} \\
& \mu^{-} e^{-} \rightarrow e^{-} e^{-} \\
& \mu^{+} e^{-} \rightarrow e^{+} e^{-} .
\end{aligned}
$$

The current bounds and possibilities for the experimental tests of muon number non-conservation is given in Table 4.

TABLE 4. Experimental Tests of Muon Number Non-Conservation.

\begin{tabular}{|c|c|c|c|}
\hline Reaction & Current Bound & Ongoing Effort & Future possibility? \\
\hline $\mathrm{R}\left(\mu^{-} N \rightarrow e^{-} N\right)$ & $<6.0 \times 10^{-13}$ & $\sim 4 \times 10^{-14}$ & $\sim 10^{-16}\left(10^{-18}\right)$ MECO \\
$\mathrm{B}\left(\mu^{+} \rightarrow e^{+} e^{-} e^{-}\right)$ & $<1.0 \times 10^{-12}$ & & \\
$\mathrm{~B}\left(\mu^{+} \rightarrow e \gamma\right)$ & & $\sim 1 \times 10^{-11}$ & $\sim \times 10^{-14}$.IEGA \\
$\mathrm{B}\left(K_{L} \rightarrow \mu e\right)$ & $<2.4 \times 10^{-11}$ & $\sim 8 \times 10^{-13}$ & BNL871 $\sim 10^{-13}$ \\
$\mathrm{~B}\left(K^{+} \rightarrow \pi^{+} \mu e\right)$ & $<2.1 \times 10^{-10}$ & $\sim 3 \times 10^{-12}$ & BNL865 $\sim 10^{-13}$ \\
$\mathrm{~B}(B \rightarrow \mu e)$ & $<5.9 \times 10^{-6}$ & Not competitive & \\
$\mathrm{B}(B \rightarrow K \mu e)$ & $<1.5 \times 10^{-5}$ & Not competitive & \\
$\mathrm{B}(Z \rightarrow \mu e)$ & $<6.0 \times 10^{-6}$ & $<10^{-13}$ from & \\
& & $\mathrm{R}\left(\mu^{-} T_{i} \rightarrow e^{-} T_{i}\right)$ & \\
\hline \hline
\end{tabular}

Using intense muon beams, it seems possible that one could push the searches for such rare reactions by four orders of magnitude. That is very impressive when one realizes that such searches are already probing one in a trillion rates.

For example, the MECO proposal at BNL aims for $2 \times 10^{-17}$ sensitivity in their search for coherent muon - electron conversion in the field of a nucleus [33]. To reach that goal requires the production, capture and stopping of muon at an unprecedented $10^{11} \frac{\mu}{\sec }$. If successful, such an effort would significantly advance the state of muon technology.

\section{SUMMARY}

High intensity muon experiments, neutrino factories, and other intermediate steps toward the muon collider are extremely important. They will greatly expand our abilities and build confidence in the credibility of high energy muon colliders.

Although a full high energy muon collider may take a considerable time to realize, intermediate steps in its direction are possible and could help facilitate the process. Muon storage ring based neutrino sources are very interesting, but require additional feasibility studies. Nore ambitious ideas for utilizing high intensity muon sources are also being explored. Indeed, if very high intensities, $\sim 10^{21} \frac{\nu}{\text { year }}$, are 
attained and nature has been kind in her neutrino mass and mixing parameters, one could envision a complete exploration of the $3 \times 3$ neutrino mixing matrix and even the detection of $\mathrm{CP}$ violation in the oscillation phenomena.

Employing an intense muon source to carry out forefront low energy research, such as the search for muon - number non - conservation, represents one interesting possibility. For example, the MECO proposal at BNL aims for $2 \times 10^{-17}$ sensitivity in their search for coherent muon - electron conversion in the field of a nucleus.

A $20 \mathrm{GeV}$ muon storage ring intense muon (neutrino) source at BNL is very interesting but expensive? An alternative source of intense muons are the conventional Horn Beams which seems to be not only competitive with the lower energy muon storage rings but also at a lower cost. For example, with the same number of proton (p) on target and same size (kTon) detector the BNL - AGS 1 Gel $\nu_{\mu}^{\text {peak }}$ Horn $\simeq 10 \mathrm{Gel}$ Muon Storage Ring (statistically if $L / E$ is fixed). Upgraded Horn facility is potentially powerful. Further $R \& D$ on $6 \times 10^{14} \mathrm{p} / \mathrm{sec}$ driver and target at BNL are important for both the muon storage ring and Horn. For more info. see references, [2] - [34].

\section{REFERENCES}

1. A.C. Melissinos unpublished note (1960). http://puhep1.princeton.edu/mumu/physics/meliss1/1.html

2. The Neutrino Factory Collaboration http://www.cap.bnl.gov/mumu/

3. http://www.awa.tohoku.ac.jp/html/KamLAND/

4. See e.g., [5] - [8]; and references therein

5. Z. Parsa, Muon Storage Rings - Neutrino Factories, in Next Generation Nucleon decay and Neutrino detector (NNN99) Workshop, SUNY, Stony Brook, September 23-25, 1999, AIP-Press (2000).

6. K.T. McDonald, ed. for the Neutrino Factory and Muon Collider Collaboration, physics/9911009, 6 Nov, 1999, and references therein; ibid, Fig 2, Private comm.

7. C.M. Ankenbrandt et al., Status of muon collider research development and future plans, Phys. Rev. ST Accel. Beams 2, 081001 (1999); refrences therein.

8. R. Palmer, C. Johnson, E. Keil, BNL-66971; http://lyoninfo.in2p3.fr/nufact99

9. http://www.cern.ch/NGS/ngs99.pdf

10. MINOS TDR, http://www.hep.anl.gov/NDK/Hypertext/minos_tdr.html

11. The MiniBooNE project: http://www.neutrino.lanl.gov/BooNE

12. The Oak Ridge Large $\nu$ Detector http://www.orau.org/orland/

13. Search for $\nu_{\mu} \rightarrow \nu_{e}$ Oscillations at CERN PS, http://chorus01.cern.ch/ pzucchel/loi/

14. BNL-P889 proposal; W.J. Marciano, Private Comm.

15. Collaboration with W.J. Marciano, to be published.

16. Y. Fukui Drawing was modified for Fig. 1. See presentation by Y. Fukui, D.Cline.

17. S. Geer, Neutrino Oscillation Rates at a Neutrino Factory, MUC0051 (1999);

18. E.g.: S. Geer, Neutrino beams from muon storage rings: Characteristics and physics potential, Phys. Rev. D 57, 6989 (1998) A. Buena, M. Campanelli, and A. Rub- 
bia, Long-baseline neutrino oscillation disappearance search using a $\nu$ beam from muon decay, hep-ph /9808485; and A. Buena, M. Campanelli, and A. Rubbia, A medium baseline search for $\nu_{\mu} \rightarrow \nu_{e}$ at a $\nu$ beam from muon decays, hep-ph/9809252; A. De Rujula, M.B. Gavela, and P. Hernandez, Nucl. Phys. B547, 21 (1999), hepph/9811390; M. Campanelli, A. Buena, and A. Rubbia, Three-family oscillations using neutrinos from muon beams at a very long hep-ph/9905420; V. Barger, S. Geer, and K. Whisnant, hep-ph/9906487; O. Yasuda, hep-ph/9910428;

I. Mocioiu and R. Shrock, hep-ph/9910554.

19. A. Blondel, Muon polarisation in the neutrino factory; http://alephwww.cern.ch/ ${ }^{\sim}$ bdl/muon/nufacpol.ps

20. V. Barger, Y.B. Dai, K. Whisnant, B.L. Young, Neutrino Mixing, CP/T Violation and Textures in Four-Neutrino Models, Phys. Rev. D 59, 113010 (1999). A. Kalliomaki, J. Mallampi, and M. Tanimoto, Search for CP Violation at a Neutrino Factory in a Four-Neutrino Model, A. Donini, M.B. Gavela, P. Hernandez, and $S$. Rigolin, Four species neutrino oscillations at $\nu$-Factory: sensitivity and $C P$ Violation, hep-ph/9910516.

21. See e.g., B.J. King, AIP Conf. Proc. 435, 334 (1998). D.A. Harris and K.S. McFarland, p. 505.

22. D. B. Cline (ed.), Physics Potential and Development of $\mu^{+} \mu^{-}$Colliders (Sausalito, CA, Nov. 1994), AIP Conf. Proc. 352 (1996).

23. The $\mu^{+} \mu^{-}$Collider Collaboration, $\mu^{+} \mu^{-}$Collider Feasibility Study, BNL-52503, FERMILAB-Conf-96/092, LBNL-38946 (July 1996).

24. Z. Parsa, ed., Future High Energy Colliders, AIP CP 397, AIP-Press, (1997).

25. Z. Parsa, Ionization cooling and Muon Dynamics, AIP CP 441, 289-294 (1997).

26. Z. Parsa, New High Intensity Muon sources and Flavor Changing Neutral Currents, World scientific Publishing, pp 147-153 (1998).

27. Kamal, B., Marciano, W., Parsa, Z., Resonant Higgs enhancement at the first muon collider, AIP CP 441, pp174- (1997); ibid, AIP 435 pp567-662 (1997).

28. Z. Parsa, Polarization and Luminosity requirements for the First Muon Collider, in AIP Conf. Proc. 472, pp. 251-259, (1998).

29. Z. Parsa, Muon Dynamics and Ionization Cooling at Muon Colliders, Procd. of EPAC98, Stockholm, Sweden, Vol 2, pp.1055- (1998).

30. C.N. Ankenbrandt et al.,Ionization Cooling Research and Development Program for a High Luminosity Muon Collider, FNAL-P904 (April 15, 1998),

31. Neutrino Factory Feasibility Studies at Fermilab, S. Geer and N. Holtkamp et al., http://www.fnal.gov/projects/muon_collider/nu_factory/.

32. Z. Parsa, New Ideas for Particle Accelerators Program Santa Barbara CA (1996); Z. Parsa, W. Marciano, W. Molzon, Y. Kuno, Y. Ogata, R. Djilkibaev et al, High Intensity Muon Source worksop, Santa Barbara CA (July 22- Aug 23, 1996).

33. see W. Molzon presentation. For Additional Theoretical discussions see presentations by W. J. Marciano.

34. Z. Parsa, New High Intensity Muon sources and Flavor Changing Neutral Currents, World scientific Publishing, pp 147-153 (1998), 